\title{
Development of a heterogeneous phantom to measure range in clinical proton therapy beams
}

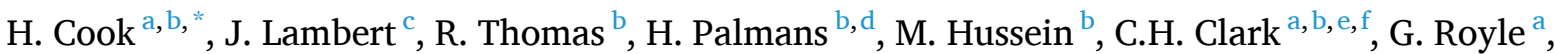 \\ $\mathrm{J}_{\text {Pettingell }}{ }^{\mathrm{c}}$, A Lourenço ${ }^{\mathrm{a}, \mathrm{b}}$ \\ ${ }^{a}$ Department of Medical Physics and Biomedical Engineering, University College London, London WC1E 6BT, United Kingdom \\ ${ }^{\mathrm{b}}$ Medical Radiation Science, National Physical Laboratory, Teddington, TW11 OLW, United Kingdom \\ ${ }^{c}$ Medical Physics Department, The Rutherford Cancer Centre South Wales, Newport NP10 8FZ, United Kingdom \\ ${ }^{\mathrm{d}}$ Medical Physics Group, MedAustron Ion Therapy Center, A-2700 Wiener Neustadt, Austria \\ ${ }^{\mathrm{e}}$ Radiotherapy Physics, University College London Hospital, NW1 2BU, United Kingdom \\ ${ }^{\mathrm{f}}$ Radiotherapy Trials Quality Assurance Group (RTTQA), Mount Vernon Cancer Centre, HA6 2RN, United Kingdom
}

\section{A R T I C L E I N F O}

\section{Keywords:}

Proton therapy

Phantom

Range verification

EBT3 film

\begin{abstract}
A B S T R A C T
Purpose: In particle therapy, determination of range by measurement or calculation can be a significant source of uncertainty. This work investigates the development of a bespoke Range Length Phantom (RaLPh) to allow independent determination of proton range in tissue. This phantom is intended to be used as an audit device. Method: RaLPh was designed to be compact and allows different configurations of tissue substitute slabs, to facilitate measurement of range using radiochromic film. Fourteen RaLPh configurations were tested, using two types of proton fluence optimised water substitutes, two types of bone substitute, and one lung substitute slabs. These were designed to mimic different complex tissue interfaces. Experiments were performed using a $115 \mathrm{MeV}$ mono-energetic scanning proton beam to investigate the proton range for each configuration. Validation of the measured film ranges was performed via Monte Carlo simulations and ionisation chamber measurements. The phantom was then assessed as an audit device, by comparing film measurements with Treatment Planning System (TPS) predicted ranges.

Results: Varying the phantom slab configurations allowed for measurable range differences, and the best combinations of heterogeneous material gave agreement between film and Monte Carlo on average within $0.2 \%$ and on average within $0.3 \%$ of ionisation chamber measurements. Results against the TPS suggest a material density override is currently required to enable the phantom to be an audit device.

Conclusion: This study found that a heterogeneous phantom with radiochromic film can provide range verification as part of a dedicated audit for clinical proton therapy beams.
\end{abstract}

\section{Introduction}

Although the finite range of protons is a key advantage of proton radiotherapy treatments compared to radiotherapy using photon beams, it is also one of the main sources of uncertainty. Due to the way protons interact with matter, varying human tissue types can result in significant changes to range within the patient. A comprehensive study of range uncertainties by Paganetti [1] identified multiple sources that contribute to this uncertainty including patient imaging, patient anatomical changes and dose calculations. Paganetti et al. reasoned the overall range uncertainty to be the combination of a $2.7 \%$ relative uncertainty and an absolute uncertainty of $1.2 \mathrm{~mm}$. As a consequence of range uncertainty, a large clinical margin is applied to proton treatment plans to guarantee full coverage of the tumour. Clinical margins vary between centres but typically a cautious $3.5 \%$ of the range plus an additional absolute value depending on the site is applied [1]. Furthermore, robust planning techniques and specific beam selection are typically applied, such as selecting multiple beam angles that do not pass through regions of daily patient variability [2]. This approach tries to ensure the clinical target is accurately covered without delivering unwanted dose to neighbouring organs at risk. Due to these constraints used in planning techniques and large clinical margins, the full potential

\footnotetext{
* Corresponding author.

E-mail address: hannah.cook@npl.co.uk (H. Cook).
} 
of proton therapy is not exploited. Therefore, range verification is clinically highly desirable to be able to establish estimates of range uncertainties in proton therapy.

A variety of approaches are in development for range verification including, in vivo beam monitoring such as through the use of Positron Emission Tomography (PET) and prompt $\gamma$ ray detection [3]. However, many of the solutions are still in the research stage and are not yet implemented in clinical centres. The use of phantoms could provide an insight into the measurement of the range within a heterogeneous scenario today. Moreover, when phantoms are used as part of independent audits, they provide dosimetric information that can offer vital awareness and understanding of any underlying issues at a radiotherapy centre. It enables reproducibility and consistency between centres for clinical trials and complex treatments [4].

For both proton reference dosimetry audits [5] and end-to-end based dosimetric audits $[4,6]$, phantoms are used that vary in shape and are made from a range of materials [7]. For proton therapy, most audit phantoms are anthropomorphic in design; including Imaging and Radiation Oncology Core's (IROC) head, paediatric spine, liver, lung and prostate phantoms as well as the CIRS head phantom $[4,8,9]$. The use of anthropomorphic phantoms for the purpose of dosimetric audit should mimic the full treatment workflow (imaging, treatment planning and dose delivery) and provide detailed information about the dose distribution by using embedded dosimeters. Although absolute dose and homogeneity of the dose is typically measured in existing audits $[4,8]$, proton range is not quantified due to the complexity of range measurements within the phantom's design. Typically, only the lateral beam profile is investigated [4]. Audit work by Taylor et al. highlighted that although proton centres are typically able to achieve acceptable dose to the tumour target, errors in range calculation impacts many phantom audits [8]. Consequently, methods to independently verify range should be an essential part of any multi-centre dosimetry audit for proton therapy. Kim et al. used a Computed Tomography (CT) calibration phantom to investigate range accuracy of a Treatment Planning System (TPS) dose algorithm and Monte Carlo simulation against measurements for eight different tissue equivalent material rods [10]. They found range prediction accuracy for heterogeneous tissues, in particular lung tissue, to be worse for the TPS compared to the Monte Carlo simulations.

As proton range estimation is a main source of uncertainty, it is important to validate range measurements in more realistic and complex phantom geometries to enable the evaluation of the full patient workflow as a means of an end-to-end audit [11]. Range measurements are typically performed with an ionisation chamber in a water phantom or with the use of array based detectors placed behind slabs of either water, tissue-substitute materials or real biological tissues [12-14]. But radiochromic film has also been previously shown to have potential as a detector for measuring proton range $[15,16]$ and the response of different types of radiochromic film (MD 55, MD 55 2, HD 810, EBT,
EBT2, EBT3, EBT XD films) in proton beams has been well documented [15-19].

In this work, radiochromic film was proposed for range measurements as it allows quick and easy determination of a full 2D distribution with high spatial resolution within one irradiation [20]. A bespoke Range Length Phantom (RaLPh) has been developed specifically to measure proton range using radiochromic film within a dosimetry audit setting. The key point of our design is a phantom which allows testing of a variety of material configurations, including bone and lung density interfaces to mimic complex clinical scenarios. This work tests whether EBT3 film can provide accurate range measurements within an heterogeneous phantom in a scanning proton therapy beam. To the best of our knowledge, this is the first phantom prototype developed for range verification within a multi-centre dosimetry audit setting.

\section{Methods and materials}

\subsection{Phantom design}

The RaLPh phantom was developed to be compact in design for simple and easy setup to enable efficient imaging and irradiation. The phantom is made of stacked $10 \times 10 \mathrm{~cm}^{2}$ slabs comprising a total length of $12.5 \mathrm{~cm}$. The first $2.5 \mathrm{~cm}$ of the phantom can be interchanged with different combinations of water equivalent material, lung, and bone substitute material plates (Fig. 1a). The remainder of the phantom is made of water equivalent material; the blocks are arranged to enable a $10 \mathrm{~cm} \times 10 \mathrm{~cm}$ piece of radiochromic film (EBT3, Gafchromic ${ }^{\mathrm{TM}}$, Ashland Inc. Wayne, NJ, henceforth referred to as EBT3 film) to be placed parallel to the beam, behind the variable section of the phantom (Fig. 1b). The phantom is encased in a PMMA frame to keep the blocks tightly packed together to avoid air gaps between them as well as to ensure setup reproducibility (Fig. 1a). Laser alignment markers were positioned on the PMMA frame sides and top of the phantom to allow for easy positioning and identification of the centre point of the phantom.

The solid water equivalent materials (SW 1471 and SW 1472, St. Bartholomew's Hospital, London, UK) used in the phantom are epoxy resin based and were previously optimised to match the proton nuclear interaction cross sections of water [13]. Both were shown to provide better water-equivalence in terms of proton fluence compared to commercial solid water equivalent materials [14]. While it was concluded that SW 1472 was the most water equivalent, it is inhomogeneous in nature due to the practicalities of mixing compounds into the epoxy resin mixture, which results in batch variability that impact the measured range and TPS calculations. Therefore, the more homogenous SW 1471 was also considered in this study, despite the fact that it was less water equivalent in terms of fluence (within $1 \%$ for a maximum depth of $10 \mathrm{~cm}$ ) [13]. Consequently, this study investigated the feasibility and limitations of using the two different SW types within RaLPh
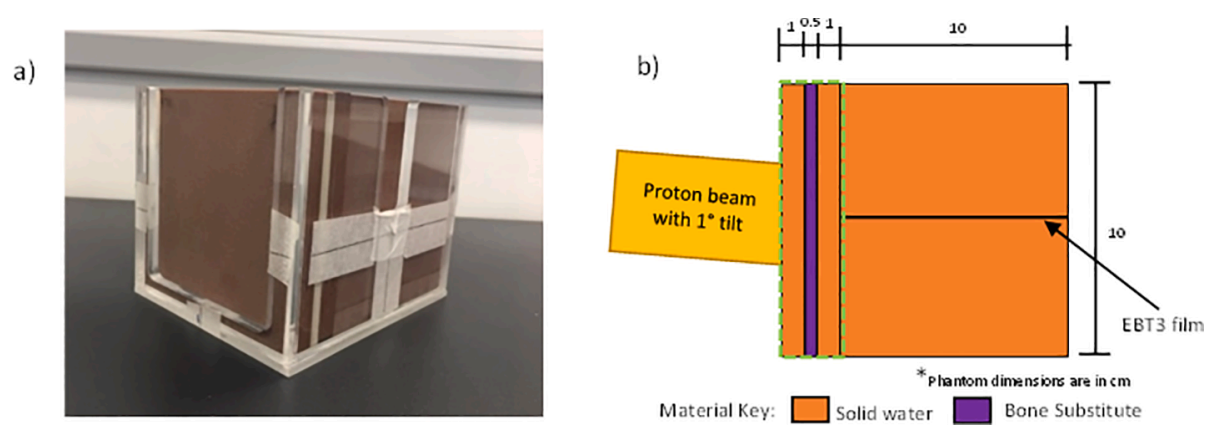

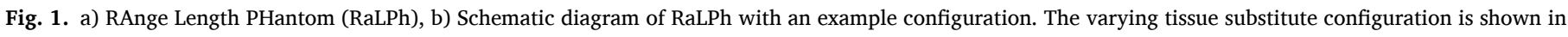

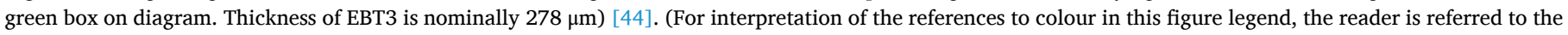
web version of this article.) 
a)

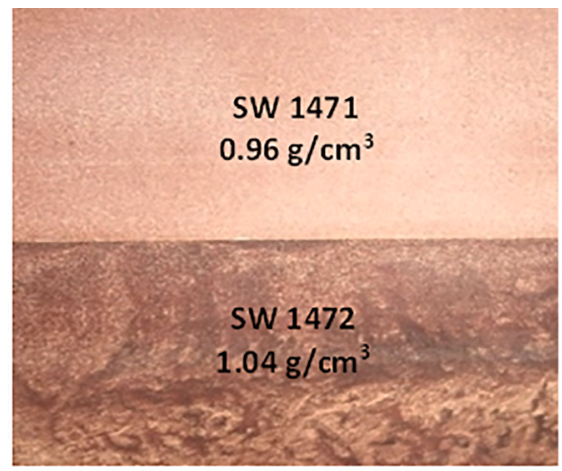

b)

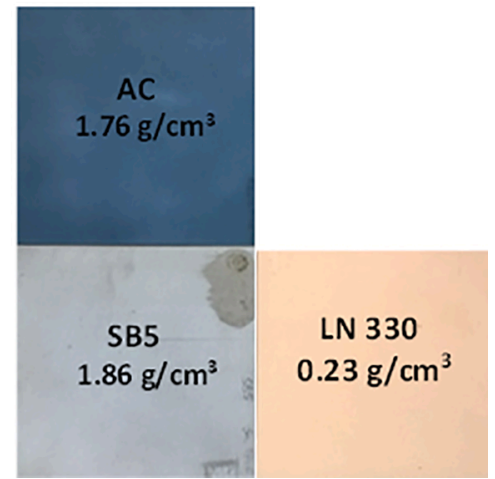

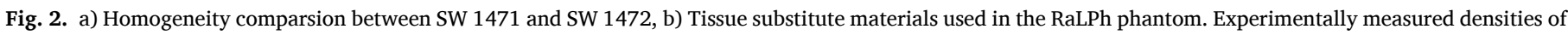

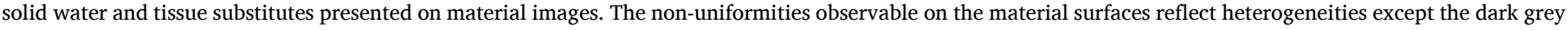
marks on SB5 which are minor surface contamination.

\section{Table 1}

Configurations tested in the variable section of the phantom. SW = Solid water (SW 1471 or SW 1472), SB5 = Hard bone, AC = Accura Bluestone and L = LN 330.

\begin{tabular}{ll}
\hline Configuration $(\mathrm{n})$ & Plates used in variable section of phantom \\
\hline 1 & $1 \mathrm{~cm} \mathrm{SW}+0.5 \mathrm{~cm} \mathrm{SW}+1 \mathrm{~cm} \mathrm{SW}$ \\
2 & $1 \mathrm{~cm} \mathrm{SW}+0.5 \mathrm{~cm} \mathrm{SB} 5+1 \mathrm{~cm} \mathrm{SW}$ \\
3 & $1 \mathrm{~cm} \mathrm{SW}+0.5 \mathrm{~cm} \mathrm{AC}+1 \mathrm{~cm} \mathrm{SW}$ \\
4 & $1 \mathrm{~cm} \mathrm{SW}+0.5 \mathrm{~cm} \mathrm{~L}+1 \mathrm{~cm} \mathrm{SW}$ \\
5 & $1 \mathrm{~cm} \mathrm{SW}+0.5 \mathrm{~cm} \mathrm{SB} 5+0.5 \mathrm{~cm} \mathrm{~L}+0.5 \mathrm{~cm} \mathrm{SW}$ \\
6 & $1 \mathrm{~cm} \mathrm{SW}+0.5 \mathrm{~cm} \mathrm{SB} 5+0.5 \mathrm{~cm} \mathrm{AC}+0.5 \mathrm{~cm} \mathrm{SW}$ \\
7 & $1 \mathrm{~cm} \mathrm{SW}+0.5 \mathrm{~cm} \mathrm{SB} 5+0.5 \mathrm{~cm} \mathrm{~L}+0.5 \mathrm{~cm} \mathrm{AC}$ \\
\hline
\end{tabular}

\section{(Fig. 2a).}

Fig. 2b shows the tissue substitute materials used in the interchangeable section of the phantom which consisted of: SW 1471 ( $\rho=$ $\left.0.96 \mathrm{~g} / \mathrm{cm}^{3}\right)$, SW $1472\left(\rho=1.04 \mathrm{~g} / \mathrm{cm}^{3}\right)$ as water substitute materials, hard bone (SB5, Leeds Test Objects (UK), $\rho=1.86 \mathrm{~g} / \mathrm{cm}^{3}$ ) and Accura Bluestone (AC, 3D Systems Corporation, $\rho=1.76 \mathrm{~g} / \mathrm{cm}^{3}$ ), as bone substitute materials, and LN 330, Leeds Test Objects (UK) (lung inhale (L), $\rho=0.23 \mathrm{~g} / \mathrm{cm}^{3}$ ) as lung substitute material $[7,21,22]$. SB5 and LN 330 are commercial tissue equivalent materials for photons while Accura Bluestone is a 3D printable material. The measured material density of the slabs was calculated by weighing them on a Mettler Toledo analytical balance (Model PG503 S) and performing length measurements with Mitutoyo Absolute IP 67 digital callipers (Model CD 8PSX).

Table 1 shows the seven different material configurations tested experimentally in the variable section of the phantom. The configurations listed in Table 1 were tested using both SW 1471 and SW 1472, hence, 14 configurations were considered in total.

\subsection{EBT3 film range measurements with RaLPh}

\subsubsection{Film handling, post-processing, and readout}

All EBT3 films were handled following radiochromic film guidelines and best procedures $[23,24]$. EBT3 films were kept in envelopes before and after irradiation to reduce light induced signal before scanning. After irradiation, all the EBT3 films were scanned using an Epson Expression 10,000 XL flatbed scanner after at least $24 \mathrm{~h}$ post proton irradiation $[20,24,25]$. The scanner bed was cleaned with ethanol before read-out of the films and warmed up by running five empty scans. The
EBT3 films were positioned in the centre of the scanner bed, scanned in the same orientation (short edge of EBT3 film perpendicular to scanning direction) to reduce scanning orientation effects and a glass compression plate was applied onto the EBT3 films before scanning [26]. One preview scan was performed on each EBT3 film before they were scanned and saved as 48-bit colour images with a resolution of $150 \mathrm{dpi}$ in a TIFF image format. EBT3 films were analysed with FilmQA Pro 2016 software (Ashland Inc. Wayne, NJ, USA).

A calibration curve is required for film measurements due to the nonlinear relationship between optical density and dose. Calibration of the EBT3 film against an ionization chamber was performed by acquiring a six-point calibration curve between 0 and $7 \mathrm{~Gy}$. A single mono-energetic layer of $115 \mathrm{MeV}$ with a $10 \times 10 \mathrm{~cm}^{2}$ field was applied vertically. The EBT3 film calibration was performed in solid water; a PTW Roos chamber 34,001 (PTW dosimetry, Freiburg, Germany) operated at -400 V was placed in a Gammex HE solid water holder (Gammex RMI GmbH, Gießen, Germany) with the EBT3 film positioned on top of the chamber and a $2 \mathrm{~cm}$ of solid water (RW3 (IBA Dosimetry, Schwarzenbruck, Germany)) as build up. The calibration was performed in the plateau region of the proton depth dose profile. A single $10 \times 10 \mathrm{~cm}^{2}$ piece of EBT3 film was used for each measurement and fiducials were marked on the EBT3 film before irradiation to align the EBT3 film and the ionisation chamber. Dose to water, $D_{w, Q}$, was derived from the ionisation chamber measurements following the recommendations of IAEA TRS 398 Code of Practice [27]:

$D_{w, Q}=M_{Q} N_{D_{w}, Q_{0}} k_{Q, Q_{0}}$

where $M_{Q}$ is the ionisation chamber reading corrected for temperature, pressure and ion recombination, $N_{D_{w}, Q_{0}}$ is the calibration coefficient for Cobalt-60 and $k_{Q, Q_{0}}$ is the beam quality correction factor. Ion recombination was determined via the two voltage method $(-100 \mathrm{~V}$ and $-400 \mathrm{~V})$ for a pulsed proton beam [27].

A central square region of interest $\left(3.4 \times 3.4 \mathrm{~cm}^{2}\right)$ was positioned directly over the projected area of the ionisation chamber's sensitive volume (with a radius of $0.78 \mathrm{~cm}$ ) for each calibration EBT3 film piece and the average scanner signal determined over this region. The relationship between the pixel values and dose was modelled using a nonlinear calibration curve $[28,29]$. The following function was fitted to the data: 
$X(D)=\frac{(a+b)}{(D-c)}$

where $X(D)$ is the average pixel value at dose $D$, and $a, b$, and $c$ are the fitted function parameters [30]. Separate film calibrations were performed for the beam tilt measurements (section 2.2.2) and for the film irradiation (section 2.2.3.) in the variable phantom configurations. The same experimental setup and film analysis procedure were used for both calibrations.

\subsubsection{Beam tilt measurements}

A study was undertaken to assess if beam tunnelling influences range measurements within the phantom. Previous research has shown that a tilt is required to film measurements when used parallel to the beam within a phantom to prevent dosimetric artefacts due to the protons tunnelling through a possible air gap $[15,17]$. An optimal degree of tilt has yet to be established, but work by Zhao and Das suggest a tilt as little as 1 degree can reduce tunnelling effects [15]. This study was performed to assess the optimal degree tilt to be used with RaLPh.

All measurements were performed at a Rutherford Cancer Centre, UK, equipped with a IBA Proteus $230 \mathrm{MeV}$ proton synchrocyclotron. RaLPh was tested using a $115 \mathrm{MeV}$ mono-energetic scanning proton beam to allow the proton beam to stop in the centre of the EBT3 film at around $10 \mathrm{~cm}$ depth in the phantom. The field size applied was a $4 \times 4$ $\mathrm{cm}^{2}$ delivering 289 spots $(0.650519 \mathrm{MU} / \mathrm{spot}$ ) resulting in a dose of $5 \mathrm{~Gy}$ at the Bragg peak. A piece of tape was placed on the front edge of the phantom so that crosshairs could be drawn to check the position of the phantom in comparison to the isocentre position (pivot point of the phantom). SW 1471 configuration 1, as described in Table 1, was used throughout the experiment. A customised RaLPh bed was developed to avoid movement of the phantom on the treatment couch when a tilt was applied. A series of different tilt angles were applied to the phantom/ couch, the gantry, or both; 0 to 5 degrees of couch tilt, 1 to 5 degrees of gantry tilt (both with 1 degree intervals) and 5 degrees of couch tilt and 5 degrees of gantry tilt (resulting in a total tilt of 10 degrees). The latter was considered as the couch tilt was limited to a maximum of 5 degrees.

\subsubsection{EBT3 film irradiations for variable phantom configurations}

For the variable phantom configuration measurements, the front face was initially setup perpendicular to the beam and the isocentre was aligned with the front edge of the phantom. A one-degree gantry tilt was applied to avoid the tunnelling of the protons through the gap containing the EBT3 film. To enable repeatable positioning after configuration changes throughout the experiment, the phantom was positioned against a treatment indexing bar to ensure the front face remained perpendicular to the beam as well as position markers were drawn around the phantom. These allowed to keep the front face of the phantom at the same angle of 89 degrees to the beam throughout the measurements. The proton beam delivery was performed with the same settings as stated in section 2.2.2. Each setup shown in Table 1 was repeated four times on separate EBT3 films.

\subsubsection{EBT3 film analysis}

Range was defined as the distal $R_{80}$ position of the proton beam where the reading (or derived dose) drops to $80 \%$ of its maximum and denoted as $R_{80}$. The $R_{80}$ range was chosen instead of the $R_{90}$ range due to it being associated with the mean projected range of the protons as well as being independent on beam's energy spread [1]. The film was cropped and aligned using FilmQA Pro software settings. The central horizontal axis was determined by calculating the distance between the top and bottom edge of the film with the cursor function. The horizontal line tool was then used to obtain the central horizontal axis optical density (OD) or dose profile (Fig. 3a and 3c) which averaged the profile over a 9pixel width. The start position of the film was defined where the first dose value was measured along the horizontal axis. The range of the proton beam was then obtained in two ways; firstly, from the OD profile
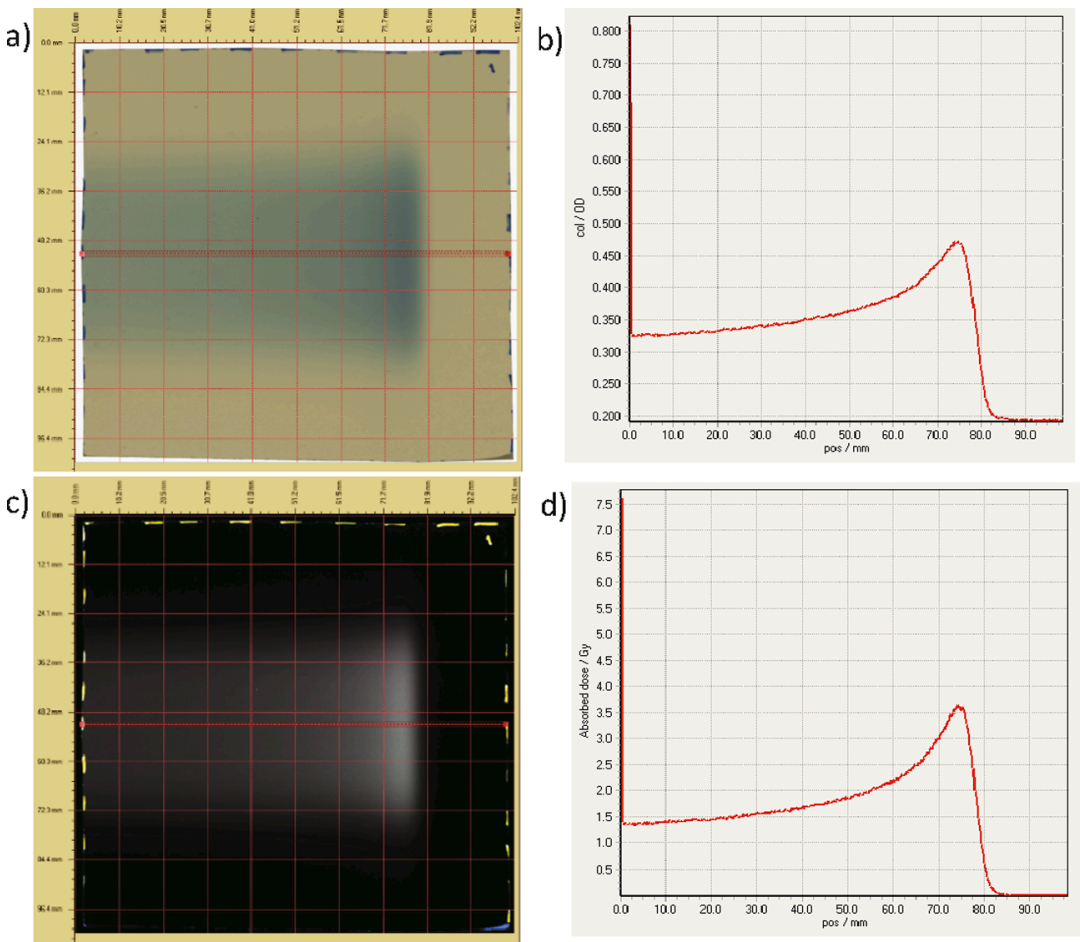

Fig. 3. a) Image of irradiated film, b) Example of the depth OD profile of film, c) Image of irradiated film after calibration is applied, d) Example of depth dose profile of film. 
on the central horizontal axis - $R_{80, \mathrm{OD}}^{\text {Film }}$ (see Fig. $3 \mathrm{~b}$ ), secondly after the film calibration was applied, from the depth dose profile on the same central horizontal axis - $R_{80, \mathrm{D}}^{\text {Film }}$ (see Fig. 3d). No correction for quenching was applied to the depth dose profiles [26]. The impact of minor positioning errors was investigated as well as its impact on the derived range. Results showed minor deviations which were within the $0.5 \%$ positioning uncertainty value given for the setup.

The position of the peak was calculated by determining a 2nd order polynomial fit of the points around the peak value and the results were then normalised to the peak value to determine the distal $R_{80}$ depth of the curve. The $R_{80}$ value was then interpolated from a linear fit around the $80 \%$ dose of the distal edge of the profile. A comparison of the $R_{80, \mathrm{OD}}^{\mathrm{Film}}$ and $R_{80, \mathrm{D}}^{\text {Film }}$ range values was carried out to investigate if a range calibration factor could be determined for EBT3 film range measurements. The use of OD only would provide improved efficiency for the audit process by not requiring a film calibration (section 2.2.1). The red channel was used for the OD analysis because it exhibits the highest absorption from the three colour channels [30]. For the dose profiles, the red channel was also used, the multichannel optimisation setting automatically corrected the red channel for film variations through use of information from the other two colour channels; this algorithm is described in Micke et al. [31]. The multichannel method has shown to reduce the uncertainty due to EBT3 film variations; including artefacts, varying thickness of the active layer, scanner nonlinearity and noise [30].

\subsection{Validation of EBT3 film measurements}

We validated the film measurements by comparing the results with ionisation chamber measurements and Monte Carlo simulations.

\subsubsection{Ionisation chamber measurements}

Ionisation chamber measurements were performed to determine the water equivalent thickness $\left(\mathrm{WET}_{\mathrm{m}}\right)$ and relative water equivalent thickness $\left(\mathrm{rWET}_{\mathrm{m}}\right.$ ) of the materials $(m)$ used within RaLPh, using equations (3) and (4), respectively [32].

$\mathrm{WET}_{\mathrm{m}}=R_{80, \mathrm{w}}^{\mathrm{IC}}-R_{80, \mathrm{w}, \mathrm{m}}^{\mathrm{IC}}$

$\mathrm{rWET}_{\mathrm{m}}=\frac{\text { WET }_{\mathrm{m}}}{t_{\mathrm{m}}}$

$R_{80, \mathrm{w}}^{\mathrm{IC}}$ corresponds to the range in water, $R_{80, \mathrm{w}, \mathrm{m}}^{\mathrm{IC}}$ is the range in water when including the material slab in front of the water phantom and $t_{\mathrm{m}}$ the thickness of the material slab.

A water phantom was setup with a large area PTW Bragg peak

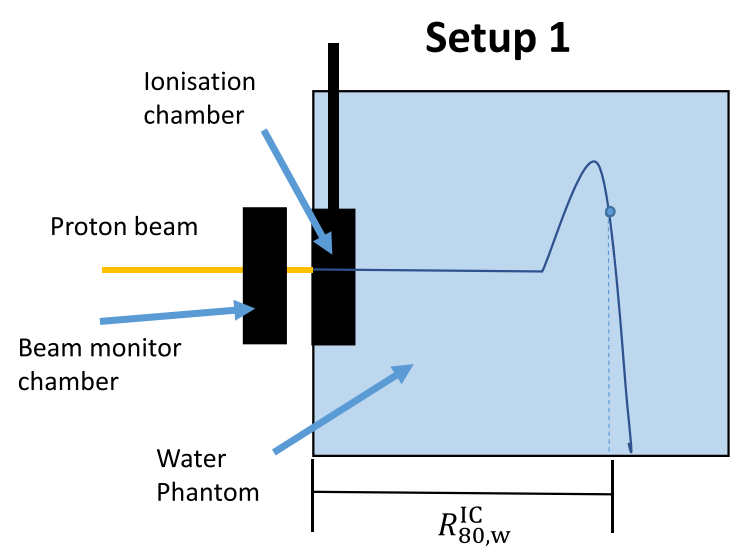

chamber 34,070 (sensitive radius $4.08 \mathrm{~cm}$ ) operated at $-400 \mathrm{~V}$ positioned $0.5 \mathrm{~mm}$ from the internal front face of the water phantom and an external PTW beam monitor chamber 7862 was positioned in front of the beam right in front of the water phantom, and its signal used for normalisation. The PTW Bragg peak chamber was moved from the front face of the water phantom in $1 \mathrm{~mm}$ steps until reaching the range in the distal fall off region of the beam so that the whole Percentage Depth Ionisation $\left(\mathrm{PDI}_{\mathrm{w}}\right)$ curve could be measured with the ionisation chamber (Fig. 4, Setup 1). The PDI measurement was repeated in smaller steps of increment $(0.2 \mathrm{~mm})$ around the Bragg peak to determine a more accurate $R_{80}$ value. The PDI curve of water was measured for a monoenergetic $115 \mathrm{MeV}$ pencil beam and then slabs of solid water $(1 \mathrm{~cm})$, lung $(0.5 \mathrm{~cm})$ and bone substitutes $(0.5 \mathrm{~cm})$ were placed individually in front of the water phantom and for each configuration the new $\mathrm{PDI}_{\mathrm{w}, \mathrm{m}}$ was measured (Fig. 4, Setup 2). PDI measurements were corrected for temperature and pressure and the Bragg Peak chamber and beam monitor ratio calculated as this accounts for any beam variations. The determination of ranges $R_{80, \mathrm{w}}^{\mathrm{IC}}$ and $R_{80, \mathrm{w}, \mathrm{m}}^{\mathrm{IC}}$ by the interpolation of the PDI followed the same approach as range determination method described in section 2.2.4.

\subsubsection{Empirical determination of solid water scaled depths}

An empirical method was used to derive the $R_{80}$ ranges for each configuration tested (Table 1) by using ionisation chamber measurements, $\mathrm{WET}_{\mathrm{m}}$ and $\mathrm{rWET}_{\mathrm{m}}$ calculations (section 2.3.1.). These independently derived ranges were then compared with the EBT3 film measurements (section 2.2.4.) to validate the EBT3 film measurements. (Further information on $\mathrm{WET}_{\mathrm{m}}$ measurements [41-43] can be found in the supplementary materials)

The range in the solid water $R_{80, s w}$ was derived using equation (5), where $R_{80, \mathrm{w}}^{\mathrm{IC}}$ was determined from the ionisation chamber measurements and $\mathrm{rWET}_{\mathrm{sw}}$ from equation (4). Equation (5) provides the range for configuration 1 from Table 1.

$R_{80, \mathrm{sw}}=\frac{R_{80, \mathrm{w}}^{\mathrm{IC}}}{\mathrm{rWET}_{\mathrm{sw}}}$

To derive the range of the other configurations (Table, 1 , configurations 2-7), firstly the solid water equivalent thickness of the material, $\mathrm{m}$, was determined via equation (6).

$t_{\mathrm{sw}-\mathrm{eq}, \mathrm{m}}=\frac{\mathrm{WET}_{\mathrm{m}}}{\mathrm{rWET}_{\mathrm{sw}}}$

The solid water equivalent thickness of the heterogeneous configurations (n) was then compared to the solid water only configuration thickness (config 1 ) to determine a solid water equivalent thickness

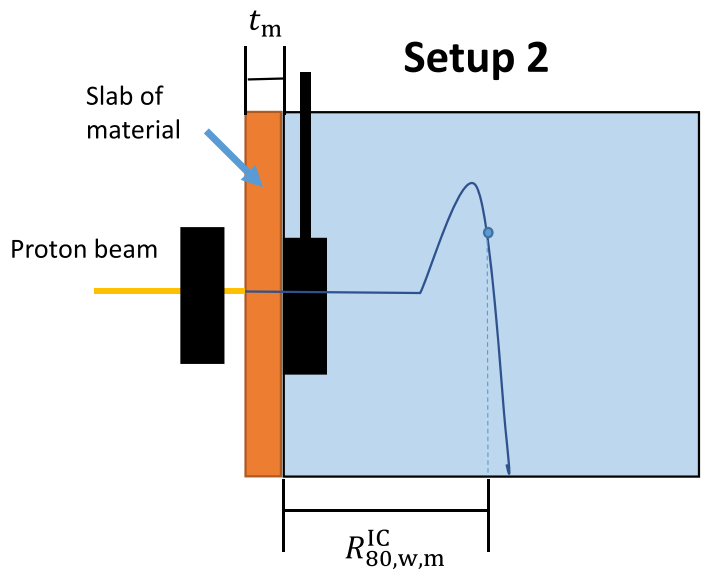

Fig. 4. Two water phantom setups (without - setup 1, left - and with - setup 2, right - RaLPh material in front of water phantom) considered in the ionisation chamber experiments and the Monte Carlo simulations. 
difference.

$\Delta t_{\mathrm{sw}-\mathrm{eq}, \mathrm{m}}^{\text {config.n }}=\mathrm{t}_{\mathrm{sw}-\mathrm{eq}, \mathrm{m}}^{\text {config.n }}-\mathrm{t}_{\mathrm{sw}}^{\text {config. } 1}$

The difference in solid water equivalent thickness was then related to the change in depth of the solid water range for the heterogeneous configuration.

$\Delta t_{\mathrm{sw}-\mathrm{eq}, \mathrm{m}}^{\mathrm{config, \textrm {n }}}=\Delta z_{\mathrm{sw}}^{\mathrm{config, \textrm {n }}}$

This enables the range determination in the heterogeneous phantom configurations to be calculated via equation (9).

$R_{80, \mathrm{sw}, \mathrm{m}}^{\mathrm{IC} \text {, config. }}=R_{80, \mathrm{sw}}-\Delta z_{\mathrm{sw}}^{\text {config.n }}$

\subsubsection{Monte Carlo calculations}

The RaLPh phantom was also modelled to derive ranges numerically by Monte Carlo simulations using the FLUKA 2011.2x.8 and Flair 2.3.0 codes [33-35]. Each simulation used $7 \times 10^{6}$ primary protons. The Monte Carlo derived ranges were then compared to EBT3 film measurements to validate them.

2.3.3.1. Beam characterisation. The beam was modelled as a monodirectional pencil beam. The mean excitation energy ( $I$-value) of water was fixed at $78 \mathrm{eV}$ [36]. The incident beam parameters (beam energy and energy spread) were tuned to achieve a match between the simulated and the $115 \mathrm{MeV}$ beam's depth dose profile measured with the ionization chamber (described in section 2.3.1). The default settings for particle therapy was used with full transport of ions while secondary electrons were set to deposit dose locally. Dose was scored with a resolution of $0.01 \mathrm{~cm}$ within a cylindrical volume with a diameter equal to the sensitive area of the PTW Bragg peak chamber. The proton transport was simulated through a water cylinder with a radius $30 \mathrm{~cm}$ and length $30 \mathrm{~cm}$.

2.3.3.2. Material characterisation. The transport of the characterised beam was simulated through a setup that replicated the experiment described in section 2.3.1. The $I$-value of the different materials listed in Table 1 were adjusted until the $R_{80}$ range matched that of the ionisation measurements in section 2.3 .1 within $0.01 \%$. It should be noted that average density measurements were used for the solid water slabs (density values can be found in Fig. 2).

2.3.3.3. Phantom simulations. The characterised beam and tuned material $I$-values were used in a box geometry surrounded by a vacuum that replicated the seven different phantom configurations for the two types of solid water. The $4 \times 4 \mathrm{~cm}^{2}$ field was simulated, and the dose was scored along the central axis of the phantom in a cylinder with a radius of $1 \mathrm{~cm}$. These results were used to determine the range in the phantom and were compared to the EBT3 film measurements.
Further information on the tuned beam characteristics and Monte Carlo derived $I$-values for the RaLPh materials can be found in the supplementary materials.

\subsection{RaLPh in a treatment planning workflow}

The phantom was tested as an audit device by acquiring a CT scan of the phantom and performing TPS calculations to determine the TPS predicted range. The TPS predicted ranges were then compared to the EBT3 film measurements.

\subsubsection{CT scanning protocol}

RaLPh was scanned with a Phillips Big Bore CT scanner. The following scanner settings were used; axial scan, tube voltage of $120 \mathrm{kV}$, voxel size of $0.117 \times 0.117 \times 0.1 \mathrm{~cm}^{3}$, average tube current of $155 \mathrm{~mA}$, helical reconstruction mode and scan reconstruction option of Brain. The 14 configurations tested were scanned in the CT scanner. Ball bearings were applied to the midpoints of the phantom surface to allow for correct alignment in the TPS.

\subsubsection{TPS calculations}

The CT data were imported into Pinnacle ${ }^{3}$ (version 16.0) to create a treatment plan and the proton range was calculated for the difference tested configurations. The plan was designed to replicate the experimental setup: a proton beam with a field size of $4 \times 4 \mathrm{~cm}^{2}$ delivering 289 spots field (0.650519 MU/spot) and the gantry was set to 89 degrees to apply the same tilt as in the experiments. A dose grid with a resolution of $0.1 \mathrm{~cm}^{3}$ was applied for each plan. The HU to stopping power calibration curve for the specific scanner used was entered into Pinnacle ${ }^{3}$ for the dose calculation. The depth dose profile was extracted as a line profile aligned with the beam axis with start and end point corresponding to the central coordinates of the phantom at the front face and the end of the phantom. The Bragg peak as well as range, $R_{80}^{\text {TPS }}$, were determined as in section 2.2.5. The density override option was also applied to the TPS plans by contouring the different materials and inputting the measured material density values (density values defined in section 2.1.). It should be noted that averaged solid water density measurements were used in the density override option. TPS range predictions were then compared to the measured range values from the film measurements.

\subsection{Summary of analysis}

Fig. 5 schematic summarises how measurements taken at the proton centre were implemented in the analysis process. $R_{80, \mathrm{D}}^{\text {Film }}$ were compared to measured and/or calculated data in order to: i) validate the EBT3 film measurements and ii) to investigate the application of the phantom in the clinic.

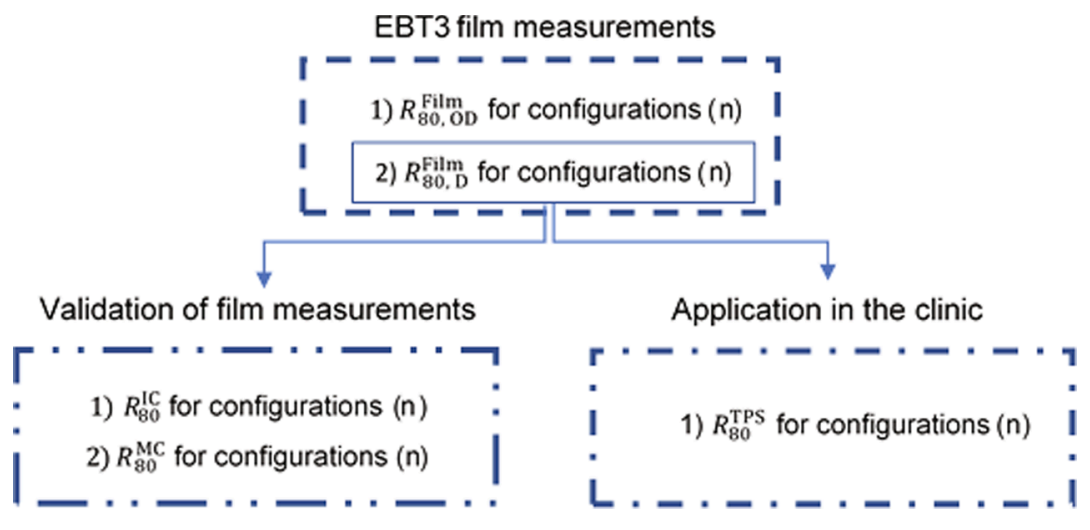

Fig. 5. Schematic of RaLPh measurements, calculations, and analysis, where $n$ is the configuration number, $R_{80, O D}^{\text {Film }}$ are the $\mathrm{R}_{80}$ measurements from the depth OD profiles of EBT3 film, $\mathrm{R}_{80, \mathrm{D}}^{\text {Film }}$ are the $\mathrm{R}_{80}$ measurements from the depth dose profiles of EBT3 film, $\mathrm{R}_{80}^{\mathrm{IC}}$ are scaled $\mathrm{R}_{80}$ values determined based on the ionisation chamber measurements, $R_{80}^{\mathrm{MC}}$ are the $\mathrm{R}_{80}$ values calculated from the depth dose profiles of Monte Carlo simulations and $R_{80}^{\text {TPS }}$ are the $\mathrm{R}_{80}$ values calculated from the TPS depth dose profiles. $\mathrm{R}_{80, \mathrm{D}}^{\mathrm{Film}}$ values were compared to other range measurements and calculated data, this is shown via the straight line box and directional arrows shown in the schematic. 


\section{Results}

\subsection{EBT3 film range measurements in RaLPh}

\subsubsection{Beam tilt measurements}

Table 2 shows the $R_{80, \mathrm{OD}}^{\text {Film }}$ and $R_{80, \mathrm{D}}^{\text {Film }}$ for the EBT3 film results for all the tilts considered in this study. The ratio between $R_{80, \mathrm{OD}}^{\text {Film }}$ and $R_{80, \mathrm{D}}^{\text {Film }}$ for the EBT3 film measurements is also provided. The results from Table 2 also show that no tunnelling effect was seen for zero-degree tilt. The results show that a range calibration factor could be applied to the depth-OD profiles as a constant value of $1.012 \pm 0.001 \mathrm{~mm}$ was found by the ratio between the OD and dose EBT3 film measurements at $R_{80}$ value.

Table 2

$R_{80}$ range measurements from tilt setups considering the $R_{80, \mathrm{OD}}^{\mathrm{Film}}$ and $R_{80 \mathrm{D}}^{\mathrm{Film}}$ ranges for the EBT3 film measurements. As well as the ratio comparison between $R_{80, \mathrm{OD}}^{\mathrm{Film}}$ and $R_{80, \mathrm{D}}^{\text {Film }}$.

\begin{tabular}{lllll}
\hline Degree of tilt & Tilt & $R_{80, \mathrm{OD}}^{\text {Film }}(\mathrm{mm})$ & $R_{80, \mathrm{D}}^{\text {Film }}(\mathrm{mm})$ & $R_{80, \mathrm{OD}}^{\text {Film }} / R_{80, \mathrm{D}}^{\text {Film }}$ \\
\hline 0 degrees & Couch tilt & 102.64 & 101.43 & 1.012 \\
1 degree & Couch tilt & 102.56 & 101.28 & 1.013 \\
2 degree & Couch tilt & 102.63 & 101.43 & 1.012 \\
4 degrees & Couch tilt & 102.70 & 101.64 & 1.010 \\
5 degrees & Couch tilt & 102.66 & 101.50 & 1.011 \\
10 degrees & Couch tilt and Gantry & 101.91 & 100.83 & 1.011 \\
5 degrees & Gantry & 102.84 & 101.70 & 1.011 \\
4 degrees & Gantry & 102.51 & 101.27 & 1.012 \\
2 degrees & Gantry & 102.57 & 101.31 & 1.012 \\
1 degree & Gantry & 103.12 & 101.80 & 1.013 \\
\hline
\end{tabular}

a)

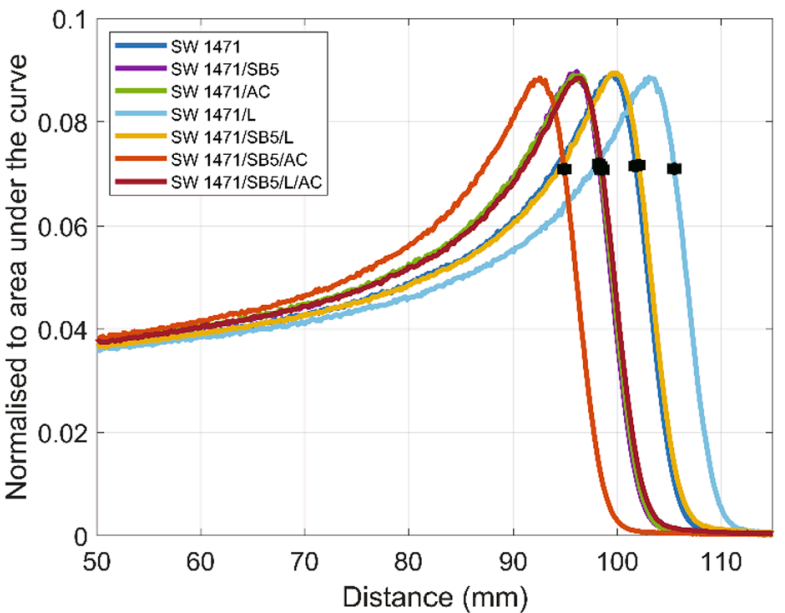

b)

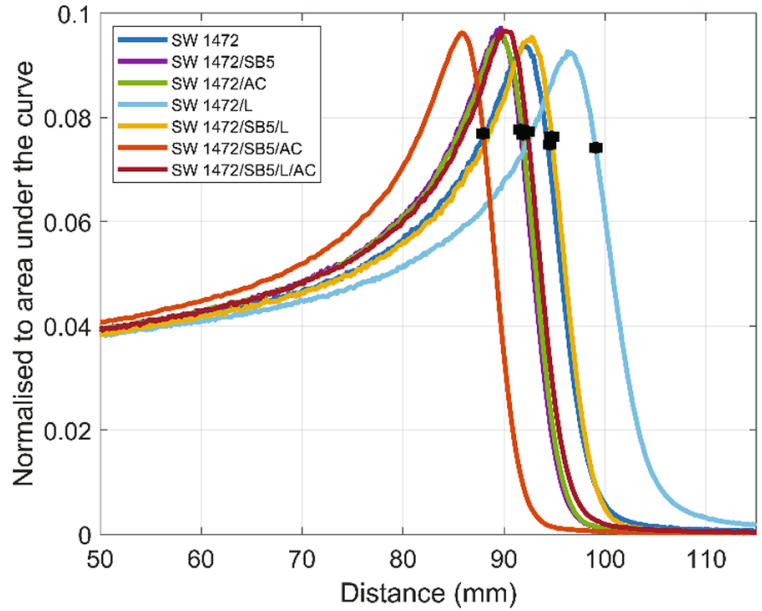

Fig. 6. Average depth-dose profile for a) SW 1471 configurations and b) SW 1472 configurations, normalised to the area under the curve. The points on the profiles are the $R_{80}$ for each configuration.

\subsubsection{EBT3 film irradiations for variable phantom configurations}

Fig. 6 shows the average depth-dose profiles for the seven SW 1471 configurations (Fig. 6a) and the seven SW 1472 configurations (Fig. 6b). The average depth-dose profiles were calculated from the four repeat measurements of each configuration. The uncertainty for each measurement was the propagation of both the $0.5 \%$ relative standard uncertainty in positioning of EBT3 film in the phantom as well as the repeatability of measurement. All uncertainties are expressed as standard uncertainties (at 1 sigma level) and are presented in the error bars shown in Fig. 6. The range of average standard uncertainty for all configurations was between 0.50 and $0.58 \%$.

Table 3 shows the $R_{80}$ values calculated from the depth-OD and depth-dose profiles for the 14 different $R a L P h$ configurations. $R_{80, D}^{\text {Film }}$ data points have been extracted from depth-dose profiles as illustrated in Fig. 6. As with the results reported in section 3.1.1., the results show that a range calibration factor value of $1.012 \pm 0.002$ was found by the ratio between the OD and dose measurements at $R_{80}$ value.

Table 3

Comparison of $\mathrm{R}_{80}$ value between depth-OD and depth-dose profiles for RaLPh phantom.

\begin{tabular}{lccl}
\hline Configuration (n) & $R_{80, \mathrm{OD}}^{\text {Film }}(\mathrm{mm})$ & $R_{80, \mathrm{D}}^{\text {Film }}(\mathrm{mm})$ & $R_{80, \mathrm{OD}}^{\text {Film }} / R_{80, \mathrm{D}}^{\text {Film }}$ \\
\hline SW 1471 & 102.91 & 101.78 & 1.011 \\
SW 1471/SB5 & 99.30 & 98.22 & 1.011 \\
SW 1471/AC & 99.49 & 98.37 & 1.011 \\
SW 1471/L & 106.90 & 105.50 & 1.013 \\
SW 1471/SB5/L & 103.10 & 102.05 & 1.010 \\
SW 1471/SB5/AC & 96.02 & 94.90 & 1.012 \\
SW 1471/SB5/L/AC & 99.70 & 98.53 & 1.012 \\
SW 1472 & 95.64 & 94.53 & 1.012 \\
SW 1472/SB5 & 92.79 & 91.61 & 1.013 \\
SW 1472/AC & 92.62 & 91.84 & 1.009 \\
SW 1472/L & 100.82 & 99.09 & 1.017 \\
SW 1472/SB5/L & 96.03 & 94.72 & 1.014 \\
SW 1472/SB5/AC & 88.97 & 87.93 & 1.012 \\
SW 1472/SB5/L/AC & 93.53 & 92.32 & 1.013 \\
\hline
\end{tabular}

\subsection{Validation of EBT3 film results}

\subsubsection{Ionisation measurements in a water phantom}

Table 4 shows the $R_{80}$ data calculated from the ionisation chamber measurements and calculated $\mathrm{WET}_{\mathrm{m}}$ and $\mathrm{rWET}_{\mathrm{m}}$ for RaLPh materials using Eqs. (3) and (4).

Table 4

Ionisation $R_{80}$ values and $\mathrm{WET}_{\mathrm{m}}$ and $\mathrm{rWET}_{\mathrm{m}}$ for RaLPh materials.

\begin{tabular}{llll}
\hline Material & $R_{80}(\mathrm{~mm})$ & WET $_{\mathrm{m}}(\mathrm{mm})$ & rWET $_{\mathrm{m}}$ \\
\hline Water & 98.85 & & \\
SB5 $(0.5 \mathrm{~cm})$ & 90.59 & 8.27 & 1.65 \\
AC $(0.5 \mathrm{~cm})$ & 90.95 & 7.90 & 1.58 \\
L $(0.5 \mathrm{~cm})$ & 97.77 & 1.09 & 0.21 \\
SW $1471(0.5 \mathrm{~cm})$ & 93.98 & 4.87 & 0.97 \\
SW $1472(0.5 \mathrm{~cm})$ & 93.50 & 5.35 & 1.07 \\
SW $1471(1.0 \mathrm{~cm})$ & 89.21 & 9.64 & 0.96 \\
SW $1472(1.0 \mathrm{~cm})$ & 88.00 & 10.85 & 1.09 \\
\hline
\end{tabular}


Table 5

Comparison of empirical calculations of proton range from the solid water scaled depth (equation (9)) with the EBT3 film range measurements $R_{80, \mathrm{D}}^{\text {Film }}$ (Table 3). Relative \% difference was calculated as follows $\left(\left(R_{80}^{\mathrm{IC}}-R_{80, D}^{\text {Film }}\right) / R_{80, \mathrm{D}}^{\text {Film }}\right) * 100$.

\begin{tabular}{lrc}
\hline Configuration (n) & $R_{80}^{\mathrm{IC}}(\mathrm{mm})$ & Relative \% Difference \\
\hline SW 1471 & 101.91 & 0.12 \\
SW 1471/SB5 & 98.39 & 0.17 \\
SW 1471/AC & 98.77 & 0.41 \\
SW 1471/L & 105.79 & 0.28 \\
SW 1471/SB5/L & 102.26 & 0.20 \\
SW 1471/SB5/AC & 95.24 & 0.36 \\
SW 1471/SB5/L/AC & 99.12 & 0.60 \\
SW 1472 & 92.38 & -2.27 \\
SW 1472/SB5 & 89.67 & -2.11 \\
SW 1472/AC & 90.02 & -1.98 \\
SW 1472/L & 96.38 & -2.73 \\
SW 1472/SB5/L & 93.67 & -1.11 \\
SW 1472/SB5/AC & 87.30 & -0.72 \\
SW 1472/SB5/L/AC & 91.30 & -1.10 \\
\hline
\end{tabular}

\subsubsection{Empirical determination of solid water scaled depths}

$\mathrm{WET}_{\mathrm{m}}$ and $\mathrm{rWET}_{\mathrm{m}}$ for the individual materials (Table 4) were used to calculate the $R_{80}^{\mathrm{IC}}$ values for the configurations by applying equations 5-7. These results are shown in Table 5 and were compared to EBT3 film measurements $\left(R_{80, \mathrm{D}}^{\text {Film }}\right)$ shown in Table 3. Results from this table show that the agreement between EBT3 film and empirical scaled depths was on average $0.3 \%$ for SW 1471 whilst for SW 1472 the agreement was within $1.7 \%$.

\subsubsection{Monte Carlo simulations}

The average $R_{80}$ percentage difference between the ionisation chamber measurements and the simulations was $0.01 \%$ for the water phantom setup. The point-to-point dose difference between the ionisation chamber measurements and simulated Bragg Peak curves was within $2 \%$. Differences were larger towards the Bragg peak due to the steep dose gradient.

Table 6 shows the simulated $R_{80}^{\mathrm{MC}}$ ranges and comparison with EBT3 film range dose measurements $\left(R_{80, \mathrm{D}}^{\text {Film }}\right)$ shown in Table 3 . The relative standard uncertainty for the repeated simulation range calculations was $0.2 \%$ for water depth-dose profiles, $0.4 \%$ for SW 1471 configurations and $0.6 \%$ for the SW 1472 configurations.

Table 6

Comparison of Monte Carlo simulated ranges with EBT3 film range measurements $R_{80, \mathrm{D}}^{\text {Film }}$ (Table 3 ). Relative $\%$ difference was calculated as follows $\left(\left(R_{80}^{\mathrm{MC}}-R_{80, \mathrm{D}}^{\text {Film }}\right) / R_{80, \mathrm{D}}^{\text {Film }}\right) * 100$.

\begin{tabular}{lrl}
\hline Configuration (n) & $R_{80}^{\mathrm{MC}}(\mathrm{mm})$ & Relative \% Difference \\
\hline SW 1471 & 101.81 & 0.03 \\
SW 1471/SB5 & 98.26 & 0.04 \\
SW 1471/AC & 98.64 & 0.27 \\
SW 1471/L & 105.72 & 0.21 \\
SW 1471/SB5/L & 102.17 & 0.12 \\
SW 1471/SB5/AC & 95.10 & 0.21 \\
SW 1471/SB5/L/AC & 99.00 & 0.48 \\
SW 1472 & 96.18 & 1.75 \\
SW 1472/SB5 & 93.12 & 1.65 \\
SW 1472/AC & 93.48 & 1.79 \\
SW 1472/L & 100.14 & 1.06 \\
SW 1472/SB5/L & 97.13 & 2.54 \\
SW 1472/SB5/AC & 90.46 & 2.88 \\
SW 1472/SB5/L/AC & 94.43 & 2.29 \\
\hline
\end{tabular}

Table 7

Comparison of TPS predicted ranged with EBT3 film range measurements $R_{80, D}^{\text {Film }}$ (Table 3). Relative \% difference was calculated as follows $\left(\left(R_{80}^{\mathrm{TPS}}-R_{80, \mathrm{D}}^{\mathrm{Film}}\right) / \mathrm{R}_{80, \mathrm{D}}^{\mathrm{Film}}\right)$ * 100.

\begin{tabular}{|c|c|c|c|c|}
\hline \multirow[t]{2}{*}{ Configuration (n) } & \multicolumn{2}{|c|}{ No density override } & \multicolumn{2}{|c|}{ Density override } \\
\hline & $R_{80}^{\mathrm{TPS}}(\mathbf{m m})$ & $\begin{array}{l}\text { Relative \% } \\
\text { Difference }\end{array}$ & $R_{80}^{\mathrm{TPS}}(\mathrm{mm})$ & $\begin{array}{l}\text { Relative \% } \\
\text { Difference }\end{array}$ \\
\hline SW 1471 & 106.31 & 4.45 & 102.25 & 0.46 \\
\hline SW 1471/SB5 & 103.74 & 5.62 & 97.95 & -0.27 \\
\hline SW 1471/AC & 103.62 & 5.34 & 98.42 & 0.05 \\
\hline SW 1471/L & 112.86 & 6.98 & 109.77 & 4.05 \\
\hline SW 1471/SB5/L & 110.62 & 8.40 & 103.96 & 1.87 \\
\hline SW 1471/SB5/AC & 100.62 & 6.03 & 96.63 & 1.82 \\
\hline SW 1471/SB5/L/AC & 106.91 & 8.51 & 101.48 & 2.99 \\
\hline SW 1472 & 99.80 & 5.57 & 95.91 & 1.46 \\
\hline SW 1472/SB5 & 96.93 & 5.81 & 92.10 & 0.53 \\
\hline SW 1472/AC & 98.48 & 7.23 & 93.04 & 1.31 \\
\hline SW 1472/L & 106.59 & 7.57 & 101.89 & 2.83 \\
\hline SW 1472/SB5/L & 103.66 & 9.44 & 98.51 & 4.00 \\
\hline SW 1472/SB5/AC & 94.51 & 7.48 & 90.58 & 3.01 \\
\hline SW 1472/SB5/L/AC & 99.20 & 7.45 & 97.77 & 5.90 \\
\hline
\end{tabular}

\subsection{RaLPh in a treatment planning workflow}

Table 7 shows the Pinnacle ${ }^{3}$ predicted $R_{80}^{\text {TPS }}$ both without and with density override for the phantom materials. The TPS results were then compared to EBT3 film measurements $\left(R_{80, \mathrm{D}}^{\text {Film }}\right)$ shown in Table 3.

\section{Discussion}

This work was completed to address the current lack of phantoms in proton beam therapy that can measure range. RaLPh is a compact, easily transportable phantom which enables quick and easy setup as well as irradiation. The beam tilt study showed that no tunnelling effect was seen for zero-degree tilt which has been observed in other film studies [17]. This may be due to the variable slab section in front of the film causing scattering effects before the beam enters the film or the phantom not having significant airgaps between slabs. The average range measured was $101.4 \mathrm{~mm}$ and the setup that showed the largest deviation of the averaged range value $(0.6 \%)$ was the setup with a 10 degrees tilt. For this setup, the film only captures the edge of the field, thus the range seen in the film is reduced to $100.83 \mathrm{~mm}$. The gantry (standard deviation $(S D)=0.27 \mathrm{~mm})$ and couch tilt $(S D=0.13 \mathrm{~mm})$ appear to have comparable range measurements, but the gantry shows a slightly larger variation in range measurements. Consequently, this study suggests a small degree tilt of 1 to 3 degrees via the couch provides the most repeatable measurements.

This work also shows the phantom design enables a range of water and tissue equivalent material configurations to be assessed via measuring range shift with EBT3 film. RaLPh shape and linear interfaces did not perturb the beam and the proton beam travels by direct transmission through the phantom to produce a sharp Bragg peak (Fig. 6). Consequently, EBT3 film can provide a simple full range measurement which allows for relative comparison of Bragg peaks and $R_{80}$ depths for a range of material configurations.

Varying the phantom plate configurations allow for measurable range differences, as shown in Fig. 6. The shift of each of the fourteen different configurations was individually determined within a range of $1 \mathrm{~cm}$. These allowed for a range of varying density material configurations to be experimentally tested and compared against TPS calculations. The EBT3 film measurements in this study showed good 
repeatability and range measurements had an average uncertainty of $0.5 \%$ when considering the relative standard uncertainty in positioning of EBT3 film in the phantom and repeatability of measurement. This work agrees with findings from previous studies which showed EBT film was capable of range measurements with a $0.5 \mathrm{~mm}$ uncertainty $[15,16]$. Our findings also suggest the possibility of using a constant range calibration factor $(1.012 \pm 0.002)$ to be applied to the depth-OD measured ranges. Typically, one of the disadvantages of using EBT3 film is that a calibration curve is required to convert OD to dose. The calibration of EBT3 film can be a time consuming process as it requires additional setup and multiple irradiation over a range of dose values; however, our results suggest a constant range calibration factor can been derived from the ratio of OD and dose values (Table 2 and 3). Hence the range calibration factor could be applied to future EBT3 film measurements for range measurements to simplify the audit process.

An extensive investigation into the validation of EBT3 film range measurements was performed. For SW 1471, the EBT3 film ranges agreed within $0.3 \%$, whilst for SW 1472 , the agreement was within $1.7 \%$ with the empirically determined scaled depths method (Table 5). With regards to the Monte Carlo simulation results, the EBT3 film range measurements were also in good agreement (within $0.2 \%$ ) for SW 1471 (Table 6), whilst for SW 1472, deviation were significant larger (1.9\%). This is due to the variability in density due to the inhomogeneities of SW 1472 which were not simulated in the Monte Carlo simulations. Although in previous work SW 1472 was found to be superior to SW 1471 , and other commercial solid water equivalent materials, in terms of proton nuclear interaction cross sections [23], homogeneity of the materials is a more important characteristic for range measurements and the results from this study support the use of SW 1471 in future range audit purposes. Overall, the validation results showed that EBT3 film can be used within a heterogeneous phantom for accurate range measurements.

In the second stage, the phantom was assessed for independent range verification capability in the clinic as part of a dosimetry audit for proton beam therapy. The phantom was applied within the treatment planning workflow by performing Pinnacle $^{3}$ dose calculations from CT scans of the phantom. Table 7 shows there was a large discrepancy between the measured EBT3 film ranges and the TPS predictions when the latter automatically assigned densities to the various materials within the phantom based on the CT calibration curve. This was due to the materials in RaLPh not being tissue equivalent in terms of photon interactions resulting in their stopping power to be wrongly derived from the stoichiometric calibration curve [37]. Some uncertainty could also be attributed to the ability to accurately distinguish each sample per voxel in the CT scan as some samples were relatively thin (5 mm thick) compared to the scan resolution of $1 \mathrm{~mm}$ per slice. Thicker slabs could reduce CT imaging uncertainty, though it should be noted that regions of thin tissue and bone can be found within the body. Nevertheless, some of this range discrepancy can be traced to the uncertainty in the TPS proton beam transport algorithm as well. When the density of the material was manually overridden (density override changes the Hounsfield unit of the material, which in turn changes the relative stopping power (RSP) in Pinnacle) in the TPS the discrepancy decreased to less than $1 \%$ if lung configurations are not considered. The lung tissue equivalent material used is very spongy in nature so there is a larger uncertainty on the measured density of this material.

Results from this study suggest that the RaLPh phantom could be used as a range audit device, if the correct material override values were provided to centres for their TPS system. This corresponds to a known issue and, for example, IROC applies a material override to some of the materials used in their Radiological Physics Center (RPC) phantoms, due to some of the phantom materials having a relative linear stopping power (RLSP) greater than 5\% from the TPS predicted values [38]. Consequently, the phantom cannot be used to validate the CT calibration curve as part of an audit. For a full treatment workflow audit, work needs to be undertaken into the development of materials that are tissue equivalent in both photon and proton beams. This will facilitate the TPS to correctly assign accurate RLSP. The full treatment workflow audit would provide a more comprehensive testing of the TPS system, including the CT calibration process. In future work, quenching corrections could also be applied to EBT3 film to achieve reference dose measurements $[15,39]$ as well as investigation into possible batch or energy dependent corrections of EBT3 film with regards to the range calibration factor. Currently, research is being undertaken to develop new tissue materials that are optimised for photon and proton therapy beams for a range of properties that will include attenuation, stopping power, scattering and nuclear interactions [40].

\section{Conclusion}

The RaLPh phantom is compact, of simple setup and provides good repeatable range measurements with EBT3 film. EBT3 film can provide repeatable simple depth dose measurements with an average relative uncertainty of $0.5 \%$ at the $R_{80}$ depth. A 1.012 range calibration factor from optical density to dose $R_{80}$ values could be applied for proton range measurements. EBT3 film measurements have been validated via Monte Carlo simulations and an empirically determined scaled depths method using ionisation chamber measurements. The phantom can be used as an audit device provided density override for the materials is used in the TPS. For the phantom to be implemented as a full treatment workflow audit device, further work needs to improve the elemental formulation of the tissue equivalent materials.

This work has shown EBT3 film to be an accurate range detector for scanning proton beam depth dose measurements within a heterogeneous phantom and that RaLPh can serve as a range audit device that can complement reference dosimetry audits and end-to-end test based dosimetric audits.

\section{Declaration of Competing Interest}

The authors declare that they have no known competing financial interests or personal relationships that could have appeared to influence the work reported in this paper.

\section{Acknowledgements}

The authors would like to thank the medical physicists and synchrocyclotron staff at The Rutherford Cancer centres for their assistance during the experiments. This work was supported by the National Measurement System of the UK's Department for Business, Energy, and Industrial Strategy. The UK National Radiotherapy Trials Quality Assurance (RTTQA) group is funded by the National Institute for Health Research (NIHR).

\section{Appendix A. Supplementary data}

Supplementary data to this article can be found online at https://doi. org/10.1016/j. ejmp.2021.11.006.

\section{References}

[1] Paganetti H. Range uncertainties in proton therapy and the role of Monte Carlo simulations. Phys Med Biol 2012;57(11):R99-117.

[2] Unkelbach J, Paganetti H. Robust Proton Treatment Planning: Physical and Biological Optimization. Semin Radiat Oncol 2018;28(2):88-96.

[3] Knopf A-C, Lomax A. In vivo proton range verification: A review. Phys Med Biol 2013;58(15):R131-60.

[4] Carlino A, Gouldstone C, Kragl G, Traneus E, Marrale M, Vatnitsky S, et al. End-toend tests using alanine dosimetry in scanned proton beams. Phys Med Biol 2018;63 (5):055001. https://doi.org/10.1088/1361-6560/aaac23.

[5] Moyers MF, Ibbott GS, Grant RL, Summers PA, Followill DS. Independent dose per monitor unit review of eight U.S.A. proton treatment facilities. Med Phys 2014;41: $1-5$. 
[6] Lewis DJ, Taylor PA, Followill DS, Sahoo N, Mahajan A, Stingo FC, et al. A New Anthropomorphic Pediatric Spine Phantom for Proton Therapy Clinical Trial Credentialing. Int J Part Ther 2018;4(4):20-7.

[7] ICRU. Tissue Substitutes in Radiation Dosimetry and Measurement Report 44; 1993.

[8] Taylor PA, Kry SF, Alvarez P, Keith T, Lujano C, Hernandez N, et al. Results From the Imaging and Radiation Oncology Core Houston's Anthropomorphic Phantoms Used for Proton Therapy Clinical Trial Credentialing. Int J Radiat Oncol Biol Phys 2016;95(1):242-8.

[9] CIRS. Proton Therapy Dosimetry Head. CIRS 2013. http://www.cirsinc.com/wpcontent/uploads/2019/04/731HN-DS-120418.pdf (accessed March 20, 2020).

[10] Kim DH, Cho S, Jo K, Shin EH, Hong CS, Han Y, et al. Proton range verification in inhomogeneous tissue: Treatment planning system vs. measurement vs. Monte Carlo simulation. PLoS ONE 2018;13:1-14.

[11] Hillbrand M, Landry G, Ebert S, Dedes G, Pappas E, Kalaitzakis G, et al. Gel dosimetry for three dimensional proton range measurements in anthropomorphic geometries. Z Med Phys 2019;29(2):162-72.

[12] Bär E, Lalonde A, Zhang R, Jee K-W, Yang K, Sharp G, et al. Experimental validation of two dual-energy CT methods for proton therapy using heterogeneous tissue samples. Med Phys 2018;45(1):48-59.

[13] Lourenço A, Shipley D, Wellock N, Thomas R, Bouchard H, Kacperek A, et al. Evaluation of the water-equivalence of plastic materials in low- and high-energy clinical proton beams. Phys Med Biol 2017;62(10):3883-901.

[14] Farr JB, Moyers MF, Allgower CE, Bues M, Hsi W-C, Jin H, et al. Clinical commissioning of intensity-modulated proton therapy systems: Report of AAPM Task Group 185. Med Phys 2021;48(1). https://doi.org/10.1002/mp. v48.110.1002/mp.14546.

[15] Zhao Li, Das IJ. Gafchromic EBT film dosimetry in proton beams. Phys Med Biol 2010;55(10):N291-301.

[16] Reinhardt S, Hillbrand M, Wilkens JJ, Assmann W. Comparison of Gafchromic EBT2 and EBT3 films for clinical photon and proton beams. Med Phys 2012;39(8): 5257-62.

[17] Piermattei A, Miceli R, Azario L, Fidanzio A, delle Canne S, De Angelis C, et al. Radiochromic film dosimetry of a low energy proton beam. Med Phys 2000;27(7): $1655-60$.

[18] Vatnitsky SM. Radiochromic film dosimetry for clinical proton beams. Appl Radiat Isot 1997;48(5):643-51.

[19] Khachonkham S, Dreindl R, Heilemann G, Lechner W, Fuchs H, Palmans H, et al. Characteristic of EBT-XD and EBT3 radiochromic film dosimetry for photon and proton beams. Phys Med Biol 2018;63(6):065007. https://doi.org/10.1088/1361 6560/aablee.

[20] Devic S, Tomic N, Lewis D. Reference radiochromic film dosimetry: Review of technical aspects. Phys Med 2016;32(4):541-56.

[21] Leeds Test Objects. Tissue Equivalent Materials: The Original Tissue Equivalents. 2014 2014:1-2. https://www.leedstestobjects.com/wp-content/uploads/TissueEquivalent-Materials-specifications.pdf?x81803.

[22] 3D Systems Corporation. Accura Bluestone (SLA). Tech Specs 2018. https:// www.3dsystems.com/materials/accura-bluestone/tech-specs (accessed March 11, 2020).

[23] Niroomand-Rad A, Blackwell CR, Coursey BM, Gall KP, Galvin JM, McLaughlin WL, et al. Radiochromic film dosimetry: recommendations of AAPM Radiation Therapy Committee Task Group 55. American Association of Physicists in Medicine. Med Phys 1998;25(11):2093-115.
[24] Bouchard H, Lacroix F, Beaudoin G, Carrier J-F, Kawrakow I. On the characterization and uncertainty analysis of radiochromic film dosimetry. Med Phys 2009;36(6Part1):1931-46.

[25] Miura H, Ozawa S, Hosono F, Sumida N, Okazue T, Yamada K, et al. Gafchromic EBT-XD film: Dosimetry characterization in high-dose, volumetric-modulated arc therapy. J Appl Clin Med Phys 2016;17(6):312-22.

[26] Marroquin EYL, Herrera González JA, Camacho López MA, Barajas JEV, GarcíaGarduño OA. Evaluation of the uncertainty in an EBT3 film dosimetry system utilizing net optical density. J Appl Clin Med Phys 2016;17(5):466-81.

[27] Andreo P. IAEA TRS-398 Absorbed Dose Determination in External Beam Radiotherapy: An International Code of Practice for Dosimetry based on Standards of Absorbed Dose to Water; 2000.

[28] Howard ME, Herman MG, Grams MP, Leal A. Methodology for radiochromic film analysis using FilmQA Pro and ImageJ. PLoS ONE 2020;15(5).

[29] Mathot M, Sobczak S, Hoornaert M. Gafchromic film dosimetry : Four years experience using FilmQA Pro software and Epson fl atbed scanners. Phys Med $2014 ; 30: 871-7$

[30] Palmer AL, Bradley D, Nisbet A. Evaluation and implementation of triple-channel radiochromic film dosimetry in brachytherapy. J Appl Clin Med Phys 2014;15(4): 280-96.

[31] Micke A, Lewis DF, Yu X. Multichannel film dosimetry with nonuniformity correction. Med Phys 2011;38(5):2523-34.

[32] Newhauser WD, Zhang R. The physics of proton therapy. Phys Med Biol 2015;80: 155-209.

[33] Battistoni G, Boehlen T, Cerutti F, Chin PW, Esposito LS, Fassò A, et al. Overview of the FLUKA code. Ann Nucl Energy 2015;82:10-8.

[34] Böhlen TT, Cerutti F, Chin MPW, Fassò A, Ferrari A, Ortega PG, et al. The FLUKA Code: Developments and challenges for high energy and medical applications. Nucl Data Sheets 2014;120:211-4.

[35] Vlachoudis V. Flair: A powerful but user friendly graphical interface for FLUKA. Proc. Int. Conf. Math. Comput. Methods React. Phys. (M\&C 2009), Saratoga Springs, New York; 2009.

[36] ICRU. Key Data For Ionizing-Radiation Dosimetry: Measurement Standards And Applications Report 90; 2016.

[37] Schneider U, Pedroni E, Lomax A. The calibration of CT Hounsfield units for radiotherapy treatment planning. Phys Med Biol 1996;41(1):111-24.

[38] Grant RL, Summers PA, Neihart JL, Blatnica AP, Sahoo N, Gillin MT, et al. Relative stopping power measurements to aid in the design of anthropomorphic phantoms for proton radiotherapy. J Appl Clin Med Phys 2014;15(2):121-6.

[39] Fiorini F, Kirby D, Thompson J, Green S, Parker DJ, Jones B, et al. Under-response correction for EBT3 films in the presence of proton spread out Bragg peaks. Phys Med 2014;30(4):454-61.

[40] Cook H, Royle G, Palmans H, Lourenço A. International Conference on Monte Carlo Techniques for Medical Applications. Simulations Improv. bone-equivalent Mater. Prot. beam Dosim; 2019.

[41] Zhang R, Newhauser WD. Calculation of water equivalent thickness of materials of arbitrary density, elemental composition and thickness in proton beam irradiation. Phys Med Biol 2009;54(6):1383-95.

[42] Gottschalk B. Comments on "Calculation of water equivalent thickness of materials of arbitrary density, elemental composition and thickness in proton beam irradiation”. Phys Med Biol 2010;55(9):L29-30.

[43] Newhauser W, Zhang R. Reply to 'Comments on "calculation of water equivalent thickness of materials of arbitrary density, elemental composition and thickness in proton beam irradiation". Phys Med Biol 2010;55(9):L31-2.

[44] Ashland Inc. GAFChromic ${ }^{\mathrm{TM}}$ EBT3 film specifications; 2014. 